\title{
Local Structure and Polar Order in Liquid N-Methyl-2-pyrrolidone (NMP)
}

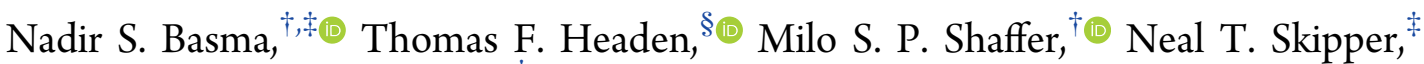 \\ and Christopher A. Howard**;
}

${ }^{\dagger}$ Department of Chemistry, Imperial College London, South Kensington, London SW7 2AZ, U.K.

${ }^{\ddagger}$ Department of Physics and Astronomy, University College London, London WC1E 6BT, U.K.

${ }^{\S}$ ISIS Neutron and Muon Source, Rutherford Appleton Laboratory, Harwell Campus, Didcot OX11 0QX, Oxfordshire, U.K.

Supporting Information

ABSTRACT: N-Methyl-2-pyrrolidone (NMP) is an exceptional solvent, widely used in industry and for nanomaterials processing. Yet despite its ubiquity, its liquid structure, which ultimately dictates its solvation properties, is not fully known. Here, neutron scattering is used to determine NMP's structure in unprecedented detail. Two dominant nearestneighbor arrangements are found, where rings are parallel or perpendicular. However, compared with related solvents, NMP has a relatively large population of parallel approaches, similar only to benzene, despite its nonaromaticity and the presence of the normally structure-reducing methyl group. This arrangement is underpinned by NMP's dipole moment, which has a profound effect on its structure: nearest-neighbor molecules arrange in an antiparallel but offset fashion. This polar-induced order extends beyond the first solvation shell, resulting in ordered trimers that reach the nanometer range. The degree of order and balance of interactions rationalize NMP's high boiling point and versatile capabilities to solvate both charged and uncharged species.

\section{INTRODUCTION}

$\mathrm{N}$-Methyl-2-pyrrolidone (NMP), $\mathrm{C}_{5} \mathrm{H}_{9} \mathrm{NO}$, is a strongly polar $(\mu=4.09 \mathrm{D})^{1}$ aprotic solvent that is a liquid at room temperature. NMP (Figure 1) exhibits a unique combination of physicochemical properties that have led to its frequent use as a solvent in a range of industrial applications. Its relatively

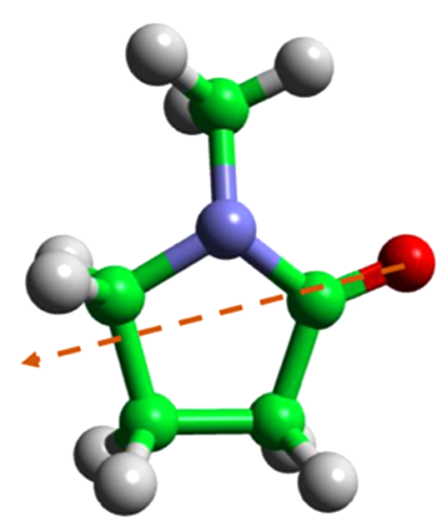

Figure 1. Structure of an $\mathrm{N}$-methyl-2-pyrroldione (NMP) molecule [green: carbon; red: oxygen; blue: nitrogen; gray: hydrogen]. The arrow indicates the polar moment of the molecule, ${ }^{18}$ directed from the carbonyl group across the ring. low volatility and affinity for aromatic hydrocarbons are exploited in petrochemical processing where it is used as an extraction medium. ${ }^{2}$ It is also commonly used for the surface treatment of textiles, resins, and plastics. ${ }^{3-6} \mathrm{NMP}$ is also an important solvent in pharmaceuticals, utilized for drug extraction and crystallization, ${ }^{7}$ and reported to increase the solubility and permeability of drugs by simultaneously acting as a co-solvent and a complexing agent. ${ }^{8,9}$ Recently, the number of scientific papers involving the use of NMP has significantly increased, ${ }^{10}$ owing to its widespread adoption as a solvent for nanomaterials processing. NMP is exceptionally successful in maintaining dispersions of nanomaterials, including graphene, ${ }^{11}$ single-walled carbon nanotubes (SWCNTs), ${ }^{12}$ and a range of other layered materials, ${ }^{13}$ following their energetic separation from one another. NMP has even been shown to dissolve these species ${ }^{14-17}$ when they are negatively charged, i.e., to form true solutions spontaneously upon contact without any external agitation.

Many efforts have been made to understand NMP's success for these processes compared with other solvents, and its ability to dissolve both ionic and neutral species. A variety of

Received: August 19, 2018

Revised: August 23, 2018

Published: August 23, 2018 
physical and chemical factors have been considered, including the possibility of solvent degradation, particularly under ultrasonication. ${ }^{19}$ The most common approach has been to compare a range of empirical parameters with related nanomaterial properties, for example, Hansen and Hildebrand solubility parameters, ${ }^{20,21}$ or matching surface energies between solvents and dispersed nanomaterials. ${ }^{22}$ However, these approaches are only partially successful, since many solvents with similar parameters are not so effective. On the other hand, it has been argued that models that treat the solvent as a uniform density dielectric continuum, and the forces between liquid dispersed nanoparticles as additive, are invalid; ${ }^{23}$ strong nanomaterials-solvent interactions can lead to dense solvation shells ${ }^{24,25}$ with potentially counterintuitive arrangements of the solvating molecules due to the balance of solvent-nanoparticle, solvent-solvent interactions and steric effects. $^{23,26}$ These effects are particularly important for understanding thermodynamic dissolution of charged nanomaterials where, similar to simple solutions, local solvent ordering around nanomaterial solutes is expected to dominate the free-energy change upon dissolution. ${ }^{23,27}$ For uncharged nanomaterials, the entropic contribution to the free energy is expected to play a critical role in determining solubilty. ${ }^{28-30}$

To fully understand NMP's solvation properties, the structure of the pure solvent must first be established. However, most of the literature surrounding NMP focuses on systems where it is mixed with another species. ${ }^{31-34}$ Studies on solid NMP (at $168 \mathrm{~K}$ ) reported the occurrence of a network of hydrogen bonding between NMP's methyl groups and the carbonyl oxygen atoms. ${ }^{35}$ In the liquid phase, NMP's structure was recently probed using X-ray scattering. The populations of the two opposite puckered conformations were calculated to be equal. ${ }^{36}$ Complementary molecular dynamics simulations, without refinement to the X-ray data, reported "part-preservation" of the hydrogen bond network reported in the crystal structure.

In recent years, there have been a number of experimental measurements of the liquid structure of simple cyclic and aromatic hydrocarbons. ${ }^{37-39}$ Within the first intersolvent coordination shell, two structural motifs are ordinarily found: parallel approaches (at slightly closer distances) and perpendicular approaches. In all cases, parallel approaches form a minor proportion, and the relative population of perpendicular arrangements is far greater. The parallel approaches at smaller separations are understood to result from aromatic ring-ring interactions, since the highest proportion is found for benzene and naphthalene. ${ }^{38,39}$ Nonaromatic systems, such as cyclohexane and cyclohexene, ${ }^{37}$ have an even lower proportion of parallel nearest neighbors. Introducing a methyl group, as in the case for toluene ${ }^{38}$ and methylcyclohexane, ${ }^{37}$ also reduces the incidence of parallel arrangements. Adding a nitrogen heteroatom to the benzene ring, as in the case of pyridine, ${ }^{39}$ induces polar ordering due to the dipole moment. NMP is a nonaromatic molecule with a five-membered ring structure, a heteroatom, and a methyl group. It has a considerably higher boiling point $\left(202{ }^{\circ} \mathrm{C}\right)$ than all of the cyclic molecules discussed above (highest boiling point is for pyridine at $115{ }^{\circ} \mathrm{C}$ ), suggesting stronger intermolecular interactions.

Here, we use advanced neutron scattering methods in combination with computational methods to uncover the detailed structure of liquid NMP. Both local structure and longer-range molecular polar-induced order are found to exist in the liquid, implicating a strong electrostatic attraction that overcomes the structurally disordering methyl group.

\section{THEORETICAL BASIS}

Following corrections, the function extracted from a neutron diffraction experiment is the total structure factor, $F(Q)$, which can be written as

$$
F(Q)=\sum_{\alpha \leq \beta}\left(2-\delta_{\alpha \beta}\right) c_{\alpha} c_{\beta} b_{\alpha} b_{\beta}\left(S_{\alpha \beta}(Q)-1\right)
$$

and is defined in terms of the magnitude of the scattering vector (or momentum change), $Q$

$$
Q=\frac{4 \pi}{\lambda} \sin \theta
$$

where $\lambda$ is the wavelength of neutrons incident onto the sample and $2 \theta$ is the scattering angle. The $S_{\alpha \beta}(Q)$ terms are FaberZiman partial structure factors, and the summation is weighted by the respective concentrations, $c_{\alpha}$ and $c_{\beta}$, and scattering lengths, $b_{\alpha}$ and $b_{\beta}$, of each atom type. The Kronecker delta function, $\delta_{\alpha \beta}$, is used to avoid double counting. The $S_{\alpha \beta}(Q)$ s contain information about correlations between the atomic species in $Q$-space, and can be related to the radial distribution functions (RDFs), $g_{\alpha \beta}(r)$, via a Fourier transformation

$$
S_{\alpha \beta}(Q)=1+4 \pi \rho \int_{0}^{\infty} r^{2}\left[g_{\alpha \beta}(r)-1\right] \frac{\sin (Q r)}{Q r} \mathrm{~d} r
$$

where $\rho$ is the atomic density of the sample.

The key aim of most structural studies of liquids is to extract the set of $g_{\alpha \beta}(r)$ functions representing real space correlations between atom pairs as a function of the separation, $r$, between them. The cumulative co-ordination number of species $\beta$ from species $\alpha$ at a distance, $r$, is given as $N_{\alpha \beta}(r)$, and can be found using

$$
N_{\alpha \beta}(r)=\int_{0}^{r} g_{\alpha \beta}(r) \rho_{\beta} \cdot 4 \pi r^{2} \mathrm{~d} r
$$

To enable the full extraction of interatomic structural correlations, or partial pair distribution functions, a series of diffraction experiments using different isotopomeric samples are required. In this way, the contrast of the total structure factor is varied, allowing for multiple diffraction patterns of a specific system to be measured. Hydrogen/deuterium isotopic substitution augmented with neutron diffraction is a powerful probe of local structure, since the largely contrasting neutron scattering lengths of hydrogen $(-3.74 \mathrm{fm})$ and deuterium $(6.88 \mathrm{fm})$ delineate the various site-site distributions necessary to describe the local interactions in a liquid.

\section{EXPERIMENTAL METHODS}

Neutron diffraction data were collected using the near and intermediate range order diffractometer (NIMROD) instrument at the ISIS spallation neutron source (Rutherford Appleton Laboratory, STFC, U.K.). ${ }^{40}$ This diffractometer was purposefully built and optimized for structural study of hydrogen-containing disordered materials. Data were measured for three samples:

(1) protiated $\mathrm{NMP}, \mathrm{C}_{4} \mathrm{H}_{9} \mathrm{NO}$;

(2) deuterated NMP, $\mathrm{C}_{4} \mathrm{D}_{9} \mathrm{NO}$; and

(3) a $1: 1$ mixture of $\mathrm{C}_{4} \mathrm{H}_{9} \mathrm{NO}$ and $\mathrm{C}_{4} \mathrm{D}_{9} \mathrm{NO}$, over the $Q$-range $0.02<Q<50 \AA^{-1}$. 
Samples were prepared and contained in flat-plate nullscattering $\mathrm{Ti}_{0.68} \mathrm{Zr}_{0.32}$ alloy cells of internal dimension $1 \mathrm{~mm} \mathrm{x}$ $35 \mathrm{~mm} \times 35 \mathrm{~mm}$. This null-scattering alloy composition ensures that the cell does not contribute any coherent neutron scattering to the measured signal. Scattering from the empty sample cells, as well as the empty instrument, was collected for data corrections. Data were then normalized, calibrated, and put on an absolute scale by comparison with the scattering from $3 \mathrm{~mm}$ vanadium plate. Particular attention was paid to correction of inelasticity effects, especially for the samples containing hydrogen. The self-scattering background and inelasticity effects were removed from the total differential scattering cross-section using an iterative method developed by Soper. $^{41,42}$

\section{COMPUTATIONAL DETAILS}

The data were modeled using the Empirical Potential Structure Refinement (EPSR) method, which in combination with neutron diffraction and isotopic substitution has successfully deduced the structures of a range of molecular liquids $37,38,43,44$ as well as solutions. ${ }^{45-49}$

A cubic box of side length 54.29 A containing 1000 NMP molecules with an atomic density of $0.10008 \AA^{-1}$ at $298 \mathrm{~K}$ was used, seeded by pairwise (Lennard-Jones and Coulomb) potentials. A combination of Lennard-Jones (LJ) and Coulomb potentials was used to build the following reference potential, $U_{\alpha \beta}(r)$, between atom pairs, $\alpha$ and $\beta$, in the molecule, represented by

$$
U_{\alpha \beta}(r)=4 \varepsilon_{\alpha \beta}\left[\left(\frac{\sigma_{\alpha \beta}}{r}\right)^{12}-\left(\frac{\sigma_{\alpha \beta}}{r}\right)^{6}\right]+\frac{1}{4 \pi \varepsilon_{0}} \frac{q_{\alpha} q_{\beta}}{r}
$$

where $\varepsilon_{\alpha \beta}$ and $\sigma_{\alpha \beta}$ are computed using the classical LorentzBerthelot mixing rules for the cross-terms and $\varepsilon_{0}$ is the permittivity of free space. The Lennard-Jones parameters, atomic masses, and charges used to seed the modeling process are summarized in Table 1 . These were obtained from the all-

Table 1. Reference Seed Potentials for All of the Labeled Atoms in the EPSR Model of Liquid NMP, Showing Lennard-Jones well-Depth Parameter, Atomic Mass, and Charge

\begin{tabular}{cccc} 
atom type & $\varepsilon / \mathrm{kJ} \mathrm{mol}^{-1}$ & $\sigma / \AA$ & $q_{\mathrm{e}}$ \\
$\mathrm{C} 1$ & 0.43932 & 3.7500 & 0.2614 \\
$\mathrm{H}_{\text {ring }}$ & 0.12552 & 2.5000 & 0.0589 \\
$\mathrm{C} 2$ & 0.27614 & 3.5000 & -0.1179 \\
$\mathrm{C} 3$ & 0.27614 & 3.5000 & -0.0696 \\
$\mathrm{~N} 1$ & 0.71128 & 3.2500 & -0.0769 \\
$\mathrm{O} 1$ & 0.87864 & 2.9600 & -0.3480 \\
$\mathrm{C} 4$ & 0.27614 & 3.5000 & -0.0613 \\
$\mathrm{H}_{\mathrm{Me}}$ & 0.12552 & 2.5000 & 0.0589 \\
\hline
\end{tabular}

atom optimized potentials for liquid simulations (OPLS-AA) force field. The labels assigned to atomic sites on the NMP molecule are shown in Figure 2.

Using the seed potentials, standard Metropolis Monte Carlo steps with traditional implementation of periodic boundary condition and the minimum image convention were used to bring the simulation to equilibrium. A smooth truncation was applied to the potential energy functions, as detailed by Soper. $^{50}$

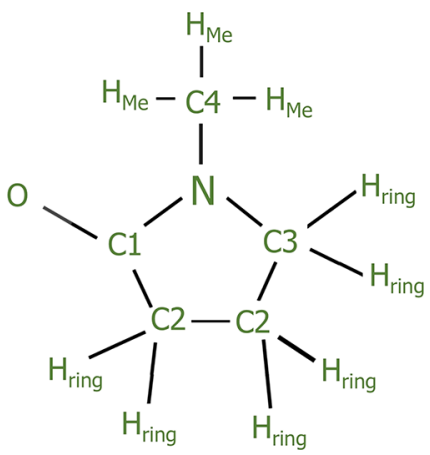

Figure 2. Atom numbering scheme for the NMP molecule in the present work. The arrow indicates the dipole moment in the molecule. $^{18}$

Once equilibrated, structure refinement was initialized. During this process, the interatomic site-site potentials are iteratively refined to drive the simulated structure toward agreement with the experimental data. This was achieved through modification of an additional empirical potential, based on the difference between measured and simulated structure factors. Once a reasonable agreement is achieved, the simulation is allowed to proceed without further perturbation of the potentials. Ensemble average structural information is then accumulated.

\section{RESULTS}

The experimental data and EPSR-refined model fits obtained for the three independent structure factors, $F(Q)$ s, as well as the total distribution functions, $f(r) \mathrm{s}$, are shown in Figure 3. A close agreement is achieved between the experimental neutron data and the EPSR-derived model. Co-ordination numbers for selected site-site correlations for the first shell were calculated and are summarized in Table 2. The RDF between the centers of geometry for NMP molecules is given in Figure 4a. It shows peaks that are well-resolved suggesting well-defined positions in relative distance, and identifies three distinct co-ordination shells localized at distances of $6.0,10.6$, and $15.7 \AA$. The integral of the $g(r)$ up to the first minimum gives the total number of molecules in the solvation shell (co-ordination number) as $\sim 13.3$. This co-ordination number is greater than that found for most aromatic molecules, ${ }^{37}$ which varies between 12.4 and 12.8 (NMP > toluene > cyclohexane/ cyclohexene > methylcyclohexane), and close to the packing of molecules in the structurally simple and tightly packed benzene (13.4).

Since site-site RDFs only give a one-dimensional representation of the liquid, using them to visualize spatial and orientational structure in three dimensions is difficult. Therefore, spatial density functions (SDFs) can be constructed to represent a three-dimensional map of the density of neighboring molecule centers around an oriented central molecule. As opposed to one-dimensional histogram binning in the case of the radial distribution function, SDFs are calculated from spatial probability densities of molecules around other molecules and amount to performing three-dimensional histogram binning. The SDF thus shows regions of space around a central molecule that are most likely to be occupied between a specified distance range. For all SDFs shown herein, the central molecule's orientation was fixed by defining two axes: an $x$-axis from atom $\mathrm{C} 3$ to $\mathrm{C} 1$ and a $y$-axis from $\mathrm{N}$ to $\mathrm{C} 4$. The $z$-axis is then formed from the cross product of the $x$ and $y$ 
(a)

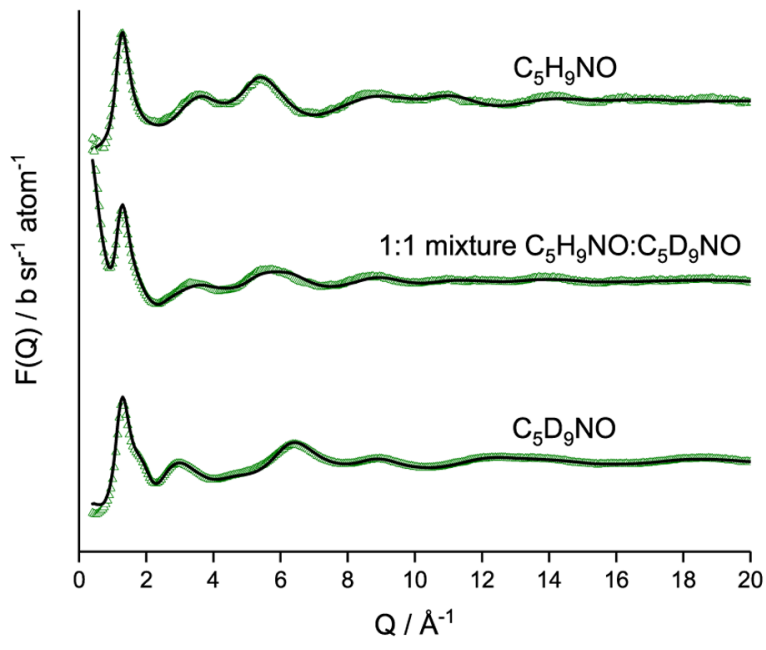

(b)

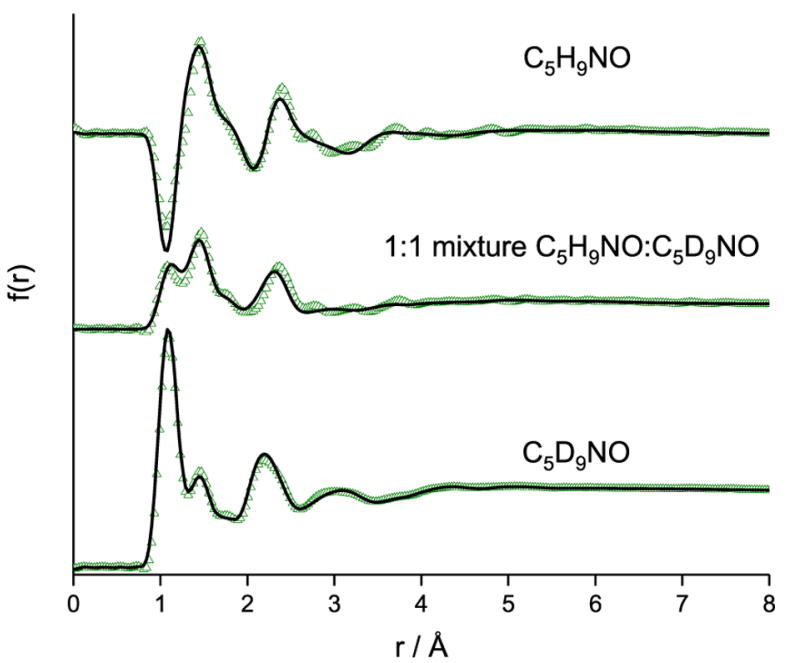

Figure 3. (a) Experimentally measured (green triangles) and EPSR-refined model fits (black lines) structure factors as a function of $Q$ for different isotopically substituted $\mathrm{N}$-methyl-2-pyrrolidone. (b) Total distribution functions in real space, $f(r)$, obtained by direct Fourier transform of the experimental structure factor data (open triangles) and the corresponding EPSR simulated functions (solid lines).

Table 2. Relevant Co-ordination Numbers Obtained by Integration of the Features in the Indicated Partial Distribution Functions

\begin{tabular}{lccc} 
correlation & $r_{\min }(\AA)$ & $r_{\max }(\AA)$ & $\mathrm{CN}$ (atoms) \\
$\mathrm{C} 1-\mathrm{H}_{\text {ring }}$ & 2.47 & 6.35 & 2.9 \\
$\mathrm{C} 1-\mathrm{H}_{\mathrm{Me}}$ & 2.47 & 6.26 & 2.9 \\
$\mathrm{C} 2-\mathrm{H}_{\mathrm{Me}}$ & 2.47 & 3.95 & 0.9 \\
& 3.95 & 5.42 & 1.4 \\
$\mathrm{~N}-\mathrm{N}$ & 3.31 & 8.05 & 4.5 \\
$\mathrm{~N}-\mathrm{O}$ & 2.72 & 4.97 & 1.5 \\
& 4.97 & 6.05 & 1.1 \\
$\mathrm{~N}-\mathrm{H}_{\text {ring }}$ & 2.39 & 4.89 & 1.5 \\
$\mathrm{~N}-\mathrm{H}_{\mathrm{Me}}$ & 2.37 & 6.39 & 3.1 \\
$\mathrm{O}-\mathrm{O}$ & 2.49 & 4.17 & 1.04 \\
$\mathrm{O}-\mathrm{H}_{\text {ring }}$ & 2.11 & 3.46 & 0.78 \\
$\mathrm{O}-\mathrm{H}_{\mathrm{Me}}$ & 2.11 & 3.50 & 0.93 \\
\hline
\end{tabular}

axes, thus effectively fixing the specified atoms as a point of reference for the central molecule.

By plotting the SDF of the centers of geometry of NMP molecules, the arrangement of the solvent molecules within this first shell (up to $8 \AA$ ) can be explored further. This is shown in Figure $4 \mathrm{~b}$ and displays a five-lobed symmetric distribution of molecules for the five-membered ring, with a small sixth lobe pointing to the oxygen atom. A continuous density band circumscribes the NMP molecule by encircling the oxygen atom and encompassing regions above and below the ring is found. There is a preference for dominant lobes to occupy the middle of the $\mathrm{C}-\mathrm{C}$ bonds, analogous to benzene ${ }^{38}$ and methylcyclohexane. ${ }^{37}$ Although the 6-fold symmetry of the most probable positions in the related methylcyclohexane molecule was found to be slightly disrupted by the presence of methyl group, the structure of NMP is significantly less disrupted by the presence of the methyl group. The lobe approaching the oxygen atom is directly aligned with this atom and at a slightly larger distance. The SDF has also been plotted from $2-4$ and 4-8 Å for clarity (Figure $4 c$ ).
Further detail about the relative arrangements of the nearestneighbor NMP can be extracted by examination of orientational correlation functions. First, we calculate the angular radial distribution functions ( $\mathrm{aRDFs}$ ), $g(r, \theta)$, where $\theta$ is the angle between the normals to the ring planes of neighboring NMP molecules, using

$$
g(r, \theta)=\frac{\Delta n(r, \theta)}{\frac{2}{3} \pi\left((r+\Delta r)^{3}-r^{3}\right) \cdot \sin \theta \cdot \Delta \theta \cdot \rho}
$$

where $\Delta n(r, \theta)$ is the number of molecules in distance range $\mathrm{r}+\Delta r$ and angle range $\theta+\Delta \theta, \rho$ is the molecular number density, and the $1 /(\sin \theta)$ factor corrects for the $\theta$-dependence of the solid angle as we integrate over the azimuthal angle. The $g(r, \theta)$ for NMP ring planes is shown in Figure 5a. At shorter distances, between 3 and $5 \AA$, two distinct peaks exist at 0 and $180^{\circ}$, indicative of ring-parallel nearest-neighbor molecules. At the longer distance range, that is, between 5 and $8 \AA$, there is a preference for perpendicular nearest neighbors $\left(\theta \approx 90^{\circ}\right)$ as shown in the SDF for this distance range in Figure 4c. Similar preferences for ring perpendicular arrangements of molecules at longer distances within the first co-ordination shell have been previously reported for a variety of other related liquids. $37,38,52$

To explore the contrasting populations of molecules in the two distances ranges seen in Figure 5a, SDFs for neighboring molecules at the shorter and longer distance ranges were also calculated (Figure 5b). The SDF isosurface (Figure 5b(i), top view) shows the preference for NMP molecules to occupy orientations parallel to the ring at the shorter distance range. On closer inspection, however, the isosurface is found to be marginally offset, toward the oxygen atom (Figure $5 b(i)$, side view); that is, the favored nearest-neighbor geometry is parallel displaced, similar to benzene. ${ }^{38,53,54}$

Quantitative analysis indicates that a notable population of molecules $(4.4 \%)$ in the first shell is oriented close to completely parallel at short distances i.e., below $5.4 \AA$. Though a small proportion, this value is greater than that found for similar cyclic hydrocarbons (between 2.3 and $3.2 \%)^{37}$ and 
(a)

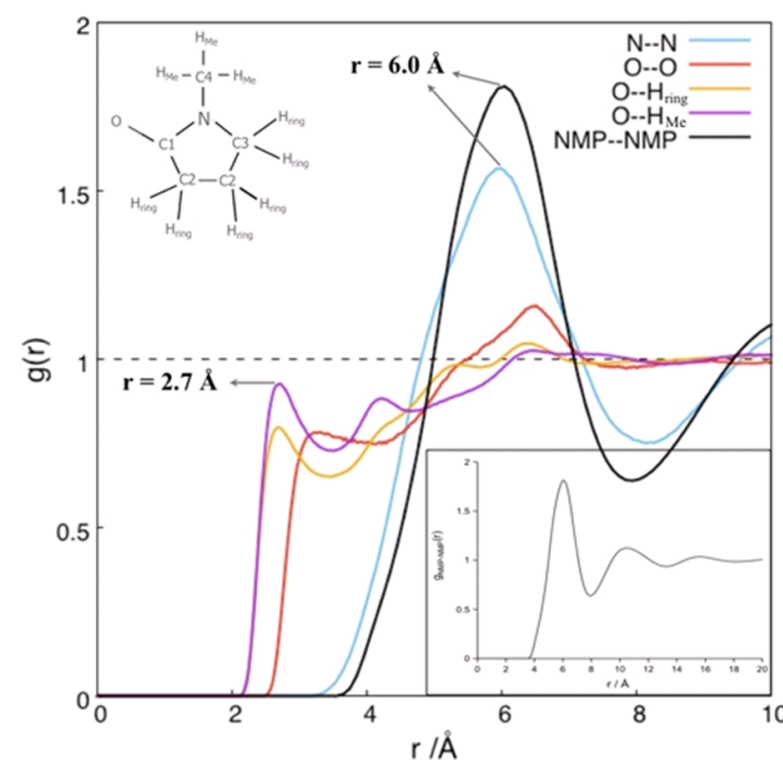

(b)

Up to $8 \AA$
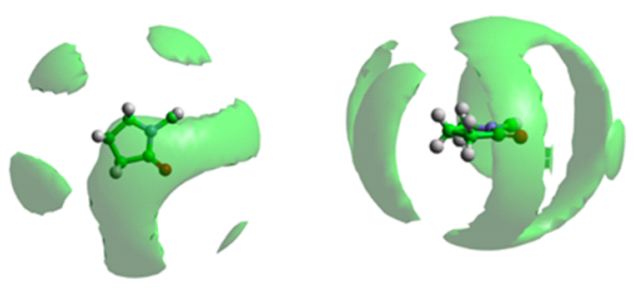

(c)
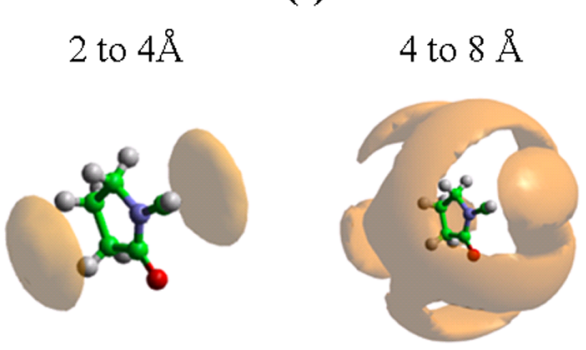

Figure 4. (a) Relevant site-site radial distribution functions, $g(r)$ s, of pure liquid $\mathrm{NMP}$. $\mathrm{H}_{\text {ring }}$ and $\mathrm{H}_{\mathrm{Me}}$ are the ring hydrogen atoms and the methyl hydrogen atoms, respectively, as shown in labeling scheme for the molecule. Inset: the NMP-NMP center of geometry $g(r)$ at room temperature, plotted up to $20 \AA$, showing three well-defined co-ordination shells. (b) Spatial probability density functions (SDFs) for liquid NMP showing the $30 \%$ most likely correlations for all molecules up to $8 \AA$ (i.e., within the first solvation shell) surrounding a central NMP species. The same data are shown from two different orientations. The three methyl hydrogens are grouped as one for a condensed view of the molecule. Surfaces are plotted using Aten. ${ }^{51}$ (c) SDFs for liquid NMP in the two distance ranges, 2-4 and 4-8 ̊.

close to the value of that for benzene (4.6\%). One interpretation is that the polar groups in NMP enhance this parallel stacking and overcome, what would normally be, the disrupting influence of the methyl group on local order.

This idea can be explored by determining whether there is any correlation of the two different populations of nearestneighbor orientations with the dipole moment of the NMP molecule, which lies very close to the direction of $\mathrm{C}=\mathrm{O}$ bond (see Figure 1). To allow for the identification of any orientational preference relative to the $\mathrm{C}=\mathrm{O}$ bonds in neighboring molecules, another aRDF was calculated, but this time for the angle between the vectors along the dipole moment of neighboring NMP molecules. This is shown in Figure 5c. Again, within the first solvation shell, there are two distinct peaks, at distances that correspond to the parallel and perpendicular approaches, revealing two populations (peaks at $\sim 4.1$ and $5.8 \AA$ ) of favorable configurations within the first nearest-neighbor zone. At the shorter distances (3-5 $\AA$ ), corresponding to parallel stacking of the molecules, the data reveal a pronounced preference for the $\mathrm{C}=\mathrm{O}$ bonds to align antiparallel to one another. For distances corresponding to the perpendicular molecules $(5-8 \AA)$, the opposite arrangement of $\mathrm{C}=\mathrm{O}$ bonds is found, that is, the polar moments in neighboring perpendicular molecules align in parallel. This ordered series of first antiparallel and then parallel orientations of the $\mathrm{C}=\mathrm{O}$ bonds is not limited to the first solvation shell, but is repeated in the second solvation shell, extending up until 13.5 $\AA$, with peaks at $\sim 8.1$ and $10.7 \AA$, demonstrating the existence of polar-ordered trimers in the liquid. Example motifs are given in Figure 5d. In methylcyclohexane, which has negligible polar moment, these parallel arrangements of nearest-neighbor molecules are absent. ${ }^{37}$ Thus, although the methyl group is sufficient to disrupt the arrangement in methylcyclohexane, this is overcome by the polar moment of the NMP molecule.

Finally, to explore hydrogen bonding present in the liquid, we study intermolecular correlations between the carbonyl oxygen and the methyl hydrogen atoms. The $\mathrm{O}-\mathrm{H}_{\text {ring }}$ and $\mathrm{O}-$ $\mathrm{H}_{\mathrm{Me}}$ RDFs (Figure $4 \mathrm{a}$ ) both show a first maximum at $2.7 \AA$, a correlation distance which occurs within the range expected for a typical hydrogen bond. ${ }^{55,56}$ The International Union of Pure and Applied Chemistry definition of a strong hydrogen bond also points to the directionality as a key defining characteristic. $^{56}$ In a strong hydrogen bond, intermolecular angle $\mathrm{X}-$ $\mathrm{H} \cdots \mathrm{Y}$ is generally linear or $180^{\circ}$, with a recommended lower limit of $110^{\circ}$ for this angle. To elucidate the directionality of this hydrogen bonding, a histogram of the intermolecular bond angles between the four atoms constituting the potentially hydrogen bond donating and accepting groups was computed. The data were then converted to a probability function shown in Figure 6.

As expected, angles below $90^{\circ}$ are highly sterically constrained due to the presence of the rest of the molecule. For angles ranging between 90 and $180^{\circ}$, there is an almost constant probability and thus there is no indication of a preferred direction, which would be expected for a strong hydrogen bond. Probabilistic analysis of the angles thus shows no indication of a strong or preferred directionality. However, despite the lack of directionality, $\mathrm{O}-\mathrm{H}_{\mathrm{Me}}$ hydrogen bonds have been observed in a variety of systems. ${ }^{57}$ The findings thus point to electrostatic weak hydrogen bonding which, though might not dominate the local structure in NMP, nevertheless provides another contributing factor, and the nondirectional hydrogen bonding ability enables a greater range of solventsolute approaches. 
(a)

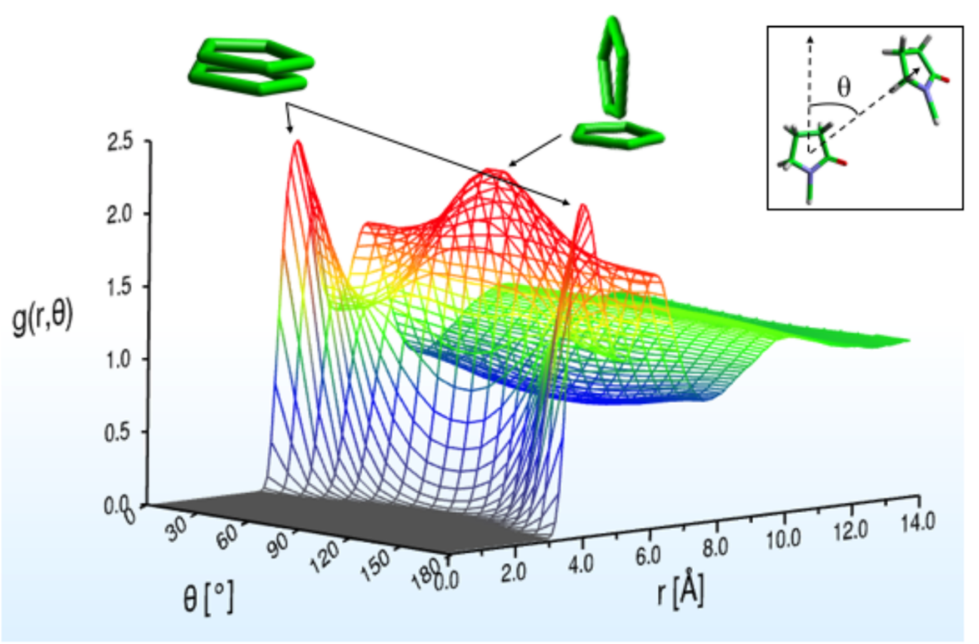

(c)

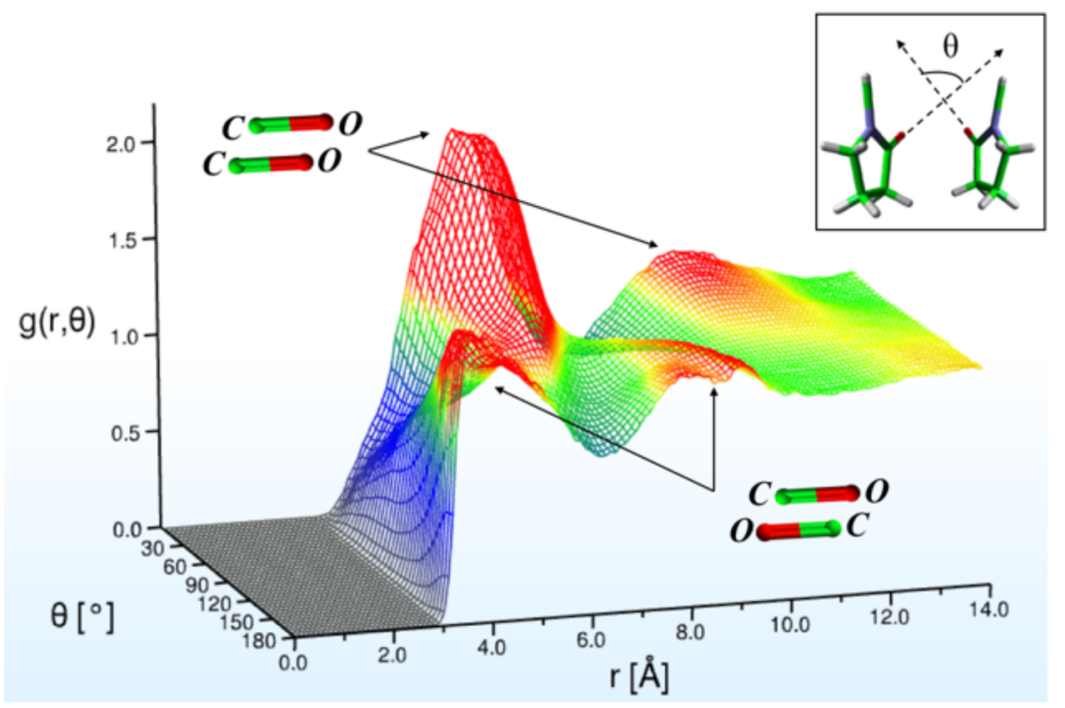

(b)

(i) 3-5 $\AA$ (top and side view)

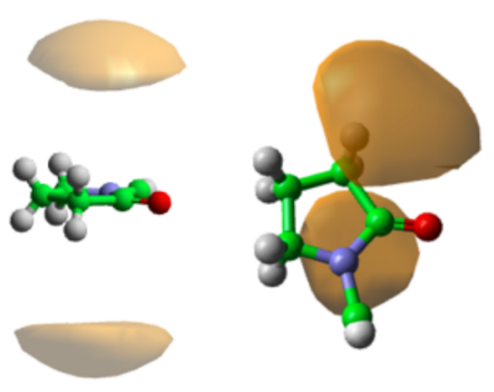

(ii) $5-8 \AA$

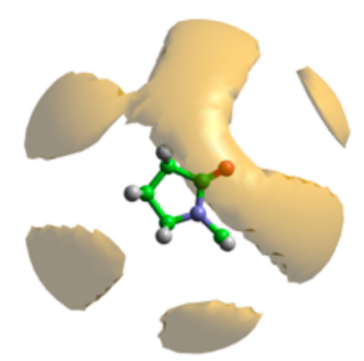

(d)

\section{$3-5 \AA$}

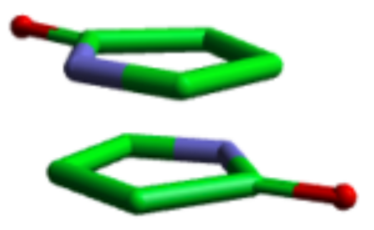

$5-8 \AA$

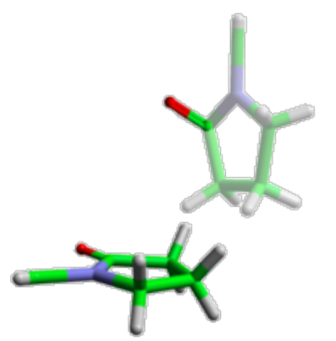

Figure 5. (a) Angular radial distribution function (aRDF) for NMP ring center-ring center, $g(r, \theta)$, where $\theta$ is the angle between the normal to the ring planes, showing preference for both parallel and perpendicular at different distance ranges. (b) Spatial probability densities for liquid NMP. The functions show the top $30 \%$ for NMP molecules in the two distance ranges, (i) $3-5 \AA$ and (ii) 5-8 $\AA$, surrounding a central NMP species. (c) aRDF, similar to (a) but with the $x$-axis defined along $\mathrm{C}=\mathrm{O}$ bond (so that the angle $\theta$ is defined as in inset) to show orientational preference of similarly aligned molecules $(\mathrm{d})$ examples of the dominant structural motifs of nearest-neighbor NMP molecules based on constraints from aRDF analysis in the distance ranges $3-5$ and 5-8 $\AA$.

\section{DISCUSSION}

NMP has long been recognized as a very important but unusual solvent. The origin of its atypical behavior has been unclear. Here, the liquid structure of NMP was investigated using neutron scattering with hydrogen/deuterium substitution in conjunction with EPSR molecular modelling simulations. This combination of experimental techniques and computational analysis allowed us to conduct a full spatial and orientational study of the solvent's structure.

Compared with related solvents, ${ }^{37-39}$ the ordering measured in liquid NMP is significantly more developed, consistent with 


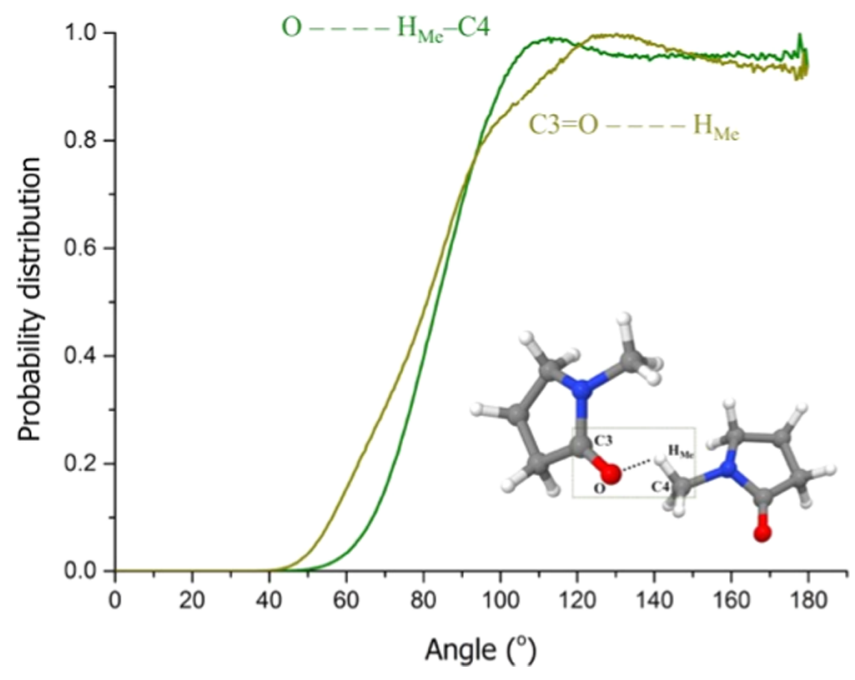

Figure 6. Probability density function showing the most probable angles for the intermolecular bond angle investigated herein. The pdf shows a wide range of conformation angles.

its considerably higher boiling point. NMP exhibits denser intermolecular packing, a greater range of local interactions and substantially longer-range molecular organization. Parallel approaches at shorter distances result from antiparallel alignment of its dipoles, similar to pyridine, ${ }^{39}$ but not only is there a greater number of parallel approaches (despite the structure-reducing methyl group), but remarkably this polarinduced order extends beyond its first solvation shell and into the nanometer range. Counterintuitively, and again uniquely compared with similar solvents, at greater distances within the nearest-neighbor shell, where the molecules are arranged perpendicularly, the dipoles align in parallel.

This intricate liquid structure rationalizes NMP's success in solvating both charged and uncharged species. ${ }^{16,58-61}$ In the case of charged nanomaterials, which react with protic solvents, such as water, ${ }^{16}$ the strong polar-induced ordering underpins aprotic NMP's effectiveness for dissolution. For both charged and uncharged nanomaterials, the range of local interactions found in NMP permits it to coordinate to itself in different configurations, thus maintaining intrasolvent bonding within and across solvation shells, ${ }^{25}$ which can be crucial for effective dissolution. ${ }^{23,26}$ NMP's effectiveness in dispersing uncharged species, such as SWCNTs, ${ }^{22}$ graphene ${ }^{62}$ and other twodimensional materials, ${ }^{63}$ can also be understood by considering the high degree of order found in the liquid. For dissolution to occur, the free energy of the solution must be lower than the combined free energy of the solvent and solute. Since pure NMP is highly structured, the entropic "cost" of solventsolute ordering is relatively low given its already low configurational entropy. This contrasts to toluene, for example, where calculations have shown that despite a favorable enthalpy change upon SWCNT dissolution, the entropic cost of the solvent reorganization is too large, explaining why SWCNTs are only sparingly soluble. ${ }^{28}$ For charged nanomaterials, the additional enthalpic gain upon solvent coordination $^{26}$ leads to spontaneous dissolution and high concentrations. ${ }^{16,24,25,64,65}$ Although $\Delta H_{\text {mix }}$ has been proposed to be the principal determining factor in the stability of uncharged solvent-SWCNT systems, ${ }^{29,30}$ the results herein thus provide a complementary explanation for the system's thermodynamic stability; i.e., the minimal overall change of
NMP's order upon solute co-ordination. Interestingly, upon the addition of $\mathrm{NaI}$ salt to a NMP/SWCNT dispersion, the SWCNTs eventually crash out of solution, ${ }^{66}$ confirming the thermodynamic dissolution of the SWCNTs and further implicating the complex balance of the different NMP/solute interactions.

It is worth noting that the modest concentrations of nanomaterials in solution mean solute-solvent interatomic correlations are of prohibitively low weighting to be measured in diffraction experiments, however, it is instructive to examine intermolecular approaches in concentrated solvent mixtures. Recent studies have highlighted the importance of preferential solute configuration in the dissolution of caffeine, ${ }^{45}$ for example, as well as the positional and orientational ordering of solvents in solute-solvent systems. ${ }^{46,47,49}$ These results further emphasize the importance of versatility for intermolecular bond formation for effective solubilization.

In summary, our findings reveal a balance of interactions in liquid NMP that are unusually well developed and dissimilar to related solvents. This intrinsic order can enable a range of local solvent-solution interactions and can serve to reduce the entropic cost accompanying on introduction of a solute, rationalizing NMP's ability to coordinate to and solvate a variety solute species, even in the absence of charge. The measured structural versatility of NMP also explains its reorganizational adaptability and enhanced ordering, for example, in vicinity of graphene. ${ }^{67,68}$

\section{ASSOCIATED CONTENT}

\section{Supporting Information}

The Supporting Information is available free of charge on the ACS Publications website at DOI: 10.1021/acs.jpcb.8b08020.

Experimental theory and methods; EPSR simulation details; co-ordination numbers; details on radial and orientational analysis; and hydrogen bonding (PDF)

\section{AUTHOR INFORMATION}

\section{Corresponding Author}

*E-mail: c.howard@ucl.ac.uk.

\section{ORCID}

Nadir S. Basma: 0000-0001-8569-5638

Thomas F. Headen: 0000-0003-0095-5731

Milo S. P. Shaffer: 0000-0001-9384-9043

Christopher A. Howard: 0000-0003-2550-0012

\section{Notes}

The authors declare no competing financial interest.

\section{ACKNOWLEDGMENTS}

We thank the Science and Technology Facilities Council (STFC) for allocated beam time at the ISIS Pulsed Neutron and Muon Source (experiment RB1010464) as well as studentship funding from the EPSRC CDT for the Advanced Characterization of Materials for N.S.B (grant EP/L015277/ 1). We thank also the computing resources provided by STFC Scientific Computing Department's SCARF cluster with which part of this work was completed.

\section{REFERENCES}

(1) Fischer, E. The Electric Moment of 1-Methylpyrrolid-2-One. J. Chem. Soc. 1955, 1382-1383. 
(2) Grishchenko, N. F.; Rogozkin, V. A.; Lastochkina, I. I.; Golubeva, K. V. Extraction of Aromatic Hydrocarbons with Mixed N-Methylpyrrolidone/glycol Solvent. Chem. Technol. Fuels Oils 1974, $10,762-764$.

(3) Comeaux, R. V.; Leighton, M. D.; Ryan, D. G. Removal of Corrodants from NMP Solvent by Contacting with Sacrificial Metal. U.S. Patent US4490240A1983.

(4) Walsh, W. C.; Waldrop, M. W.; James, L. E.; Monahan, W. Method of Cleaning Polymer Residues with NMP. U.S. Patent US5698045A1996.

(5) Bushnell, J. D. Lube Extraction with NMP/phenol/water Mixtures. U.S. Patent US4013549A1974.

(6) Iwasaki, T.; Horikoshi, K.; Yoshiji, Y. Process for Recovering NMethylpyrrolidone by Plural Distillations. U.S. Patent US4976825A1989.

(7) Xu, W.-L.; Mao, F.; Zhao, H.-K.; Wang, Y.; Wang, J. Solubility of Anthracene in N,N-Dimethyformamide, N,N-Dimethylacetamide, and N-Methyl-2-Pyrrolidone. J. Chem. Eng. Data 2007, 52, 553-554.

(8) Uch, A. S.; Hesse, U.; Dressman, J. B. Use of 1-MethylPyrrolidone as a Solubilizing Agent for Determining the Uptake of Poorly Soluble Drugs. Pharm. Res. 1999, 16, 968-971.

(9) Lee, P. J.; Langer, R.; Shastri, V. P. Role of N-Methyl Pyrrolidone in the Enhancement of Aqueous Phase Transdermal Transport. J. Pharm. Sci. 2005, 94, 912-917.

(10) Ashcroft, C. P.; Dunn, P. J.; Hayler, J. D.; Wells, A. S. Survey of Solvent Usage in Papers Published in Organic Process Research \& Development 1997-2012. Org. Process Res. Dev. 2015, 19, 740-747.

(11) Hernandez, Y.; Nicolosi, V.; Lotya, M.; Blighe, F. M.; Sun, Z.; De, S.; McGovern, I. T.; Holland, B.; Byrne, M.; Gun'Ko, Y. K.; et al. High-Yield Production of Graphene by Liquid-Phase Exfoliation of Graphite. Nat. Nanotechnol. 2008, 3, 563-568.

(12) Hasan, T.; Scardaci, V.; Tan, P. H.; Rozhin, A. G.; Milne, W. I.; Ferrari, A. C. Stabilization And "debundling" of Single-Wall Carbon Nanotube Dispersions in N-Methyl-2-Pyrrolidone (NMP) by Polyvinylpyrrolidone (PVP). J. Phys. Chem. C 2007, 111, 1259412602.

(13) Coleman, J. N.; Lotya, M.; O’Neill, A.; Bergin, S. D.; King, P. J.; Khan, U.; Young, K.; Gaucher, A.; De, S.; Smith, R. J.; et al. TwoDimensional Nanosheets Produced by Liquid Exfoliation of Layered Materials. Science 2011, 331, 568-571.

(14) Pénicaud, A.; Poulin, P.; Derré, A.; Anglaret, E.; Petit, P. Spontaneous Dissolution of a Single-Wall Carbon Nanotube Salt. J. Am. Chem. Soc. 2005, 127, 8-9.

(15) Vallés, C.; Drummond, C.; Saadaoui, H.; Furtado, C. A.; He, M.; Roubeau, O.; Ortolani, L.; Monthioux, M.; Pénicaud, A. Solutions of Negatively Charged Graphene Sheets and Ribbons. J. Am. Chem. Soc. 2008, 130, 15802-15804.

(16) Cullen, P. L.; Cox, K. M.; Bin Subhan, M. K.; Picco, L.; Payton, O. D.; Buckley, D. J.; Miller, T. S.; Hodge, S. A.; Skipper, N. T.; Tileli, V.; et al. Ionic Solutions of Two-Dimensional Materials. Nat. Chem. 2017, 9, 244-249.

(17) Miller, T. S.; Suter, T. M.; Telford, A. M.; Picco, L.; Payton, O. D.; Russell-Pavier, F.; Cullen, P. L.; Sella, A.; Shaffer, M. S. P.; Nelson, J.; et al. Single Crystal, Luminescent Carbon Nitride Nanosheets Formed by Spontaneous Dissolution. Nano Lett. 2017, 17, 58915896.

(18) Aparicio, S.; Alcalde, R.; Dávila, M. J.; García, B.; Leal, J. M. Measurements and Predictive Models for the N -Methyl-2pyrrolidone/Water/Methanol System. J. Phys. Chem. B 2008, 112, 11361-11373.

(19) Yau, H. C.; Bayazit, M. K.; Steinke, J. H. G.; Shaffer, M. S. P. Sonochemical Degradation of N-Methylpyrrolidone and Its Influence on Single Walled Carbon Nanotube Dispersion. Chem. Commun. 2015, 51, 16621-16624.

(20) Detriche, S.; Zorzini, G.; Colomer, J.-F.; Fonseca, A.; Nagy, J. B. Application of the Hansen Solubility Parameters Theory to Carbon Nanotubes. J. Nanosci. Nanotechnol. 2008, 8, 6082-6092.
(21) Cheng, Q.; Debnath, S.; O’Neill, L.; Hedderman, T. G.; Gregan, E.; Byrne, H. J. Systematic Study of the Dispersion of SWNTs in Organic Solvents. J. Phys. Chem. C 2010, 114, 4857-4863.

(22) Bergin, S. D.; Sun, Z.; Streich, P.; Hamilton, J.; Coleman, J. N. New Solvents for Nanotubes: Approaching the Dispersibility of Surfactants. J. Phys. Chem. C 2010, 114, 231-237.

(23) Silvera Batista, C. A.; Larson, R. G.; Kotov, N. A. Nonadditivity of Nanoparticle Interactions. Science 2015, 350, No. 1242477.

(24) Howard, C. A.; Wasse, J. C.; Skipper, N. T.; Thompson, H.; Soper, A. K. The Solvation Structure of Fulleride C605- Anions in Potassium Ammonia Solution. J. Phys. Chem. C 2007, 111, 56405647.

(25) Howard, C.; Thompson, H.; Wasse, J. C.; Skipper, N. T. Formation of Giant Solvation Shells around Fulleride Anions in Liquid Ammonia. J. Am. Chem. Soc. 2004, 126, 13228-13229.

(26) Howard, C.; Skipper, N. T. Computer Simulations of Fulleride Anions in Metal-Ammonia Solutions. J. Phys. Chem. B 2009, 113, 3324-3332.

(27) Murrell, J. N.; Jenkins, A. D. In Properties of Liquids and Solutions; John, N., Aubrey, D., Eds.; Wiley \& Sons, 1994.

(28) Grujicic, M.; Cao, G.; Roy, W. N. Atomistic Simulations of the Solubilization of Single-Walled Carbon Nanotubes in Toluene. J. Mater. Sci. 2004, 39, 2315-2325.

(29) Bergin, S. D.; Nicolosi, V.; Streich, P. V.; Giordani, S.; Sun, Z.; Windle, A. H.; Ryan, P.; Niraj, N. P. P.; Wang, Z.-T. T.; Carpenter, L.; et al. Towards Solutions of Single-Walled Carbon Nanotubes in Common Solvents. Adv. Mater. 2008, 20, 1876-1881.

(30) Bergin, S. D.; Sun, Z.; Rickard, D.; Streich, P. V.; Hamilton, J. P.; Coleman, J. N. Multicomponent Solubility Parameters for SingleWalled Carbon Nanotube-Solvent Mixtures. ACS Nano 2009, 3, 2340-2350.

(31) Carver, T. J.; Drew, M. G. B.; Mark Rodger, P. Molecular Dynamics Calculations of N-Methylpyrrolidone in Liquid Water. Phys. Chem. Chem. Phys. 1999, 1, 1807-1816.

(32) Karpenko, O. B.; Trachevskij, V. V.; Filonenko, O. V.; Lobanov, V. V.; Avdeev, M. V.; Tropin, T. V.; Kyzyma, O. A.; Snegir, S. V.; et al. NMR Study of Non-Equilibrium State of Fullerine C60 in N-Methyl2-Pyrrolidone. Ukr. J. Phys. 2012, 57, 860-863.

(33) Usula, M.; Porcedda, S.; Mocci, F.; Gontrani, L.; Caminiti, R.; Cesare Marincola, F. NMR, Calorimetry, and Computational Studies of Aqueous Solutions of N -Methyl-2-Pyrrolidone. J. Phys. Chem. B 2014, 118, 10493-10502.

(34) Usula, M.; Mocci, F.; Cesare Marincola, F.; Porcedda, S.; Gontrani, L.; Caminiti, R. The Structural Organization of N-Methyl2-Pyrrolidone + Water Mixtures: A Densitometry, X-Ray Diffraction, and Molecular Dynamics Study. J. Chem. Phys. 2014, 140, No. 124503.

(35) Müller, G.; Lutz, M.; Harder, S. Methyl Group ConformationDetermining Intermolecular C-H...O Hydrogen Bonds: Structure of N -Methyl-2-Pyrrolidone. Acta Crystallogr., Sect. B: Struct. Sci. 1996, $52,1014-1022$.

(36) Gontrani, L.; Caminiti, R. The Structure of Liquid N-Methyl Pyrrolidone Probed by X-Ray Scattering and Molecular Simulations. J. Chem. Phys. 2012, 136, No. 074505.

(37) Falkowska, M.; Bowron, D.; Manyar, H.; Hardacre, C.; Youngs, T. Neutron Scattering of Aromatic and Aliphatic Liquids. ChemPhysChem 2016, 17, 2043-2055.

(38) Headen, T. F.; Howard, C. A.; Skipper, N. T.; Wilkinson, M. A.; Bowron, D. T.; Soper, A. K. Structure of $\Pi-\pi$ Interactions in Aromatic Liquids. J. Am. Chem. Soc. 2010, 132, 5735-5742.

(39) Headen, T. F.; Cullen, P. L.; Patel, R.; Taylor, A.; Skipper, N. $\mathrm{T}$. The Structures of Liquid Pyridine and Naphthalene: The Effects of Heteroatoms and Core Size on Aromatic Interactions. Phys. Chem. Chem. Phys. 2018, 20, 2704-2715.

(40) Bowron, D. T.; Soper, A. K.; Jones, K.; Ansell, S.; Birch, S.; Norris, J.; Perrott, L.; Riedel, D.; Rhodes, N. J.; Wakefield, S. R.; et al. NIMROD: The Near and InterMediate Range Order Diffractometer of the ISIS Second Target Station. Rev. Sci. Instrum. 2010, 81, No. 033905. 
(41) Soper, A. K. Inelasticity Corrections for Time-of-Flight and Fixed Wavelength Neutron Diffraction Experiments. Mol. Phys. 2009, 107, 1667-1684.

(42) Soper, A. K. The Radial Distribution Functions of Water as Derived from Radiation Total Scattering Experiments: Is There Anything We Can Say for Sure? ISRN Phys. Chem. 2013, 2013, 1-67. (43) Soper, A. K. The Radial Distribution Functions of Water and Ice from 220 to $673 \mathrm{~K}$ and at Pressures up to $400 \mathrm{MPa}$. Chem. Phys. 2000, 258, 121-137.

(44) McLain, S. E.; Soper, A. K.; Luzar, A. Orientational Correlations in Liquid Acetone and Dimethyl Sulfoxide: A Comparative Study. J. Chem. Phys. 2006, 124, No. 074502.

(45) Tavagnacco, L.; Brady, J. W.; Bruni, F.; Callear, S.; Ricci, M. A.; Saboungi, M. L.; Cesàro, A. Hydration of Caffeine at High Temperature by Neutron Scattering and Simulation Studies. J. Phys. Chem. B 2015, 119, 13294-13301.

(46) Johnston, A. J.; Zhang, Y.; Busch, S.; Pardo, L. C.; Imberti, S.; McLain, S. E. Amphipathic Solvation of Indole: Implications for the Role of Tryptophan in Membrane Proteins. J. Phys. Chem. B 2015, 119, 5979-5987.

(47) Shephard, J. J.; Callear, S. K.; Imberti, S.; Evans, J. S. O.; Salzmann, C. G. Microstructures of Negative and Positive Azeotropes. Phys. Chem. Chem. Phys. 2016, 18, 19227-19235.

(48) Ingram, D. J.; Headen, T. F.; Skipper, N. T.; Callear, S. K.; Billing, M.; Sella, A. Dihydrogen: Vs. Hydrogen Bonding in the Solvation of Ammonia Borane by Tetrahydrofuran and Liquid Ammonia. Phys. Chem. Chem. Phys. 2018, 20, 12200-12209.

(49) Henao, A.; Johnston, A. J.; Guàrdia, E.; McLain, S. E.; Pardo, L. C. On the Positional and Orientational Order of Water and Methanol around Indole: A Study on the Microscopic Origin of Solubility. Phys. Chem. Chem. Phys. 2016, 18, 23006-23016.

(50) Soper, A. K. Tests of the Empirical Potential Structure Refinement Method and a New Method of Application to Neutron Diffraction Data on Water. Mol. Phys. 2001, 99, 1503-1516.

(51) Youngs, T. G. A. Aten - An Application for the Creation, Editing, and Visualization of Coordinates for Glasses, Liquids, Crystals, and Molecules. J. Comput. Chem. 2010, 31, 639-648.

(52) Bochynski, Z.; Drozdowski, H. X-Ray Diffraction Investigation of Intermolecular Ordering in Liquid Benzene, Cyclohexane and NHexane. Acta Phys. Slovaca 1999, 49, 409-418.

(53) Sherrill, C. D.; Takatani, T.; Hohenstein, E. G. An Assessment of Theoretical Methods for Nonbonded Interactions: Comparison to Complete Basis Set Limit Coupled-Cluster Potential Energy Curves for the Benzene Dimer, the Methane Dimer, Benzene-Methane, and Benzene-H2S. J. Phys. Chem. A 2009, 113, 10146-10159.

(54) Sinnokrot, M. O.; Sherrill, C. D. High-Accuracy Quantum Mechanical Studies of $\pi$ - $\pi$ Interactions in Benzene Dimers. J. Phys. Chem. A 2006, 110, 10656-10668.

(55) Jeffrey, G. A. An Introduction to Hydrogen Bonding; Oxford University Press, 1997.

(56) Arunan, E.; Desiraju, G. R.; Klein, R. A.; Sadlej, J.; Scheiner, S.; Alkorta, I.; Clary, D. C.; Crabtree, R. H.; Dannenberg, J. J.; Hobza, P.; et al. Defining the Hydrogen Bond: An Account (IUPAC Technical Report). Pure Appl. Chem. 2011, 83, 1619-1636.

(57) Desiraju, G.; Steiner, T. The Weak Hydrogen Bond; Oxford University Press, 2001.

(58) Catheline, A.; Ortolani, L.; Morandi, V.; Melle-Franco, M.; Drummond, C.; Zakri, C.; Pénicaud, A. Solutions of Fully Exfoliated Individual Graphene Flakes in Low Boiling Point Solvents. Soft Matter 2012, 8, 7882 .

(59) Catheline, A.; Vallés, C.; Drummond, C.; Ortolani, L.; Morandi, V.; Marcaccio, M.; Iurlo, M.; Paolucci, F.; Pénicaud, A. Graphene Solutions. Chem. Commun. 2011, 47, 5470.

(60) Ausman, K.; Piner, R.; Lourie, O.; et al. Organic Solvent Dispersions of Single-Walled Carbon Nanotubes: Toward Solutions of Pristine Nanotubes. J. Phys. Chem. B 2000, 104, 8911-8915.

(61) Furtado, C. A.; Kim, U. J.; Gutierrez, H. R.; Pan, L.; Dickey, E. C.; Eklund, P. C. Debundling and Dissolution of Single-Walled
Carbon Nanotubes in Amide Solvents. J. Am. Chem. Soc. 2004, 126, 6095-6105.

(62) Coleman, J. N. Liquid-Phase Exfoliation of Nanotubes and Graphene. Adv. Funct. Mater. 2009, 19, 3680-3695.

(63) Backes, C.; Higgins, T. M.; Kelly, A.; Boland, C.; Harvey, A.; Hanlon, D.; Coleman, J. N. Guidelines for Exfoliation, Characterization and Processing of Layered Materials Produced by Liquid Exfoliation. Chem. Mater. 2017, 29, 243-255.

(64) Pénicaud, A.; Dragin, F.; Pécastaings, G.; He, M.; Anglaret, E. Concentrated Solutions of Individualized Single Walled Carbon Nanotubes. Carbon 2014, 67, 360-367.

(65) Buckley, D. J.; Hodge, S. A.; De Marco, M.; Hu, S.; Anthony, D. B.; Cullen, P. L.; McKeigue, K.; Skipper, N. T.; Shaffer, M. S. P.; Howard, C. A. Trajectory of the Selective Dissolution of Charged Single-Walled Carbon Nanotubes. J. Phys. Chem. C 2017, 121, 21703-21712.

(66) Frolov, A. I.; Arif, R. N.; Kolar, M.; Romanova, A. O.; Fedorov, M. V.; Rozhin, A. G. Molecular Mechanisms of Salt Effects on Carbon Nanotube Dispersions in an Organic Solvent (N-Methyl-2-Pyrrolidone). Chem. Sci. 2012, 3, 541-548.

(67) Arunachalam, V.; Vasudevan, S. Graphene-Solvent Interactions in Nonaqueous Dispersions: 2D ROESY NMR Measurements and Molecular Dynamics Simulations. J. Phys. Chem. C 2018, 122, $1881-1888$.

(68) Terrones, J.; Kiley, P. J.; Elliott, J. A. Enhanced Ordering Reduces Electric Susceptibility of Liquids Confined to Graphene Slit Pores. Sci. Rep. 2016, 6, No. 27406. 